\title{
Spectroscopic and Photochemical Properties of Secondary Brown Carbon from Aqueous Reactions of Methylglyoxal
}

\author{
Aaron W. Harrison,* Amanda M. Waterson, and Warren J. De Bruyn \\ Schmid College of Science and Technology, Chapman University, Orange, CA 98266, U.S.A.
}

\section{Supporting Information}

Index

I. BrC Synthesis

II. BrC Photolysis Rates and Estimated Atmospheric Half-lives

III. PARAFAC Analysis

a. Excitation and Emission Spectra of PARAFAC Components

b. Fluorophore Photolytic Half-lives

IV. Proposed Structures from Mass Spectra

*Author to whom correspondence should be addressed: aharrison@ chapman.edu 


\section{BrC Synthesis}

To synthesize the $\mathrm{BrC}$ samples studied in the present work, $0.2 \mathrm{M}$ stock solutions of aqueous methylglyoxal (MG) were mixed at equal proportion $(10 \mathrm{~mL}+10 \mathrm{~mL})$ with $0.2 \mathrm{M}$ stock solutions of $\left(\mathrm{NH}_{4}\right)_{2} \mathrm{SO}_{4}$ (AS) or $\mathrm{NH}_{2} \mathrm{CH}_{3} \cdot \mathrm{HCl}$ (MA) to give a final concentration of $\sim 100 \mathrm{mM}$ of each reactant. These concentrations are higher than typical MG concentrations found in cloud water $(\sim 1-100 \mu \mathrm{M})^{1}$ which could lead to increased browning and larger mass absorption coefficients in the near UV. However, the concentrations used here are similar to precursor concentrations used in previous studies to facilitate more direct comparison. ${ }^{2}$ Regardless of the initial concentration, the concentration of the reaction solution will increase as water evaporates during the drying step (discussed below).

Reaction solutions were aged for $\sim 1$ day in the dark at room temperature before being transferred to a beaker for drying in air over the course of $\sim 2$ days in a fume hood. The aging in the condensed phase for $\sim 1$ day allowed further time for $\mathrm{MG}$ to reach its hydration equilibria after being diluted from the $40 \mathrm{wt} \%$ solution purchased from Sigma-Aldrich. ${ }^{3}$ No significant browning of the solution was observed over this timescale. After drying, the MG+AS BrC samples were a brown crystalline solid while the dried MG+MA BrC samples led to a brown viscous, paste. These solids were weighed and re-dissolved in the same initial volume of Milli-Q water $(20 \mathrm{~mL})$ to make a stock solution of each of the $\mathrm{BrC}$ samples. To prevent saturation, these stock solutions required further dilution for spectroscopic measurements to obtain absorbance values $<2$. Control syntheses were conducted by drying the reactants independently and did not result in browning of any of the MG, AS, or MA reactants.

There have been a variety of Imine $\mathrm{BrC}$ synthesis methods reported in the literature including aging aqueous mixtures over weeks to months, ${ }^{4,5}$ drying in nitrogen, ${ }^{6-8}$ and using a rotary evaporator. ${ }^{2,9,10}$ Although these reaction mixtures will brown over time in the aqueous phase, imine formation is driven by dehydration reactions (see Scheme 1) and evaporation considerably accelerates these browning reactions. ${ }^{11}$ In addition, the products formed under evaporative conditions are more atmospherically relevant as they can be formed rapidly in a single cloud processing event. ${ }^{2}, 12$ We felt the above synthesis method best simulated chromophore formation from aqueous aerosol and cloud water processing in the atmosphere by allowing the reactants to dry at ambient temperature and pressure rather than using a rota-vap. 
II. BrC Photolysis Rates and Estimated Atmospheric Photolytic Half-lives

Table S1. MG + AS and MG + MA BrC Photolysis Rates and Estimated Atmospheric Halflives (see Sec. 3.2 of the manuscript)

\begin{tabular}{|c|c|}
\hline $\mathbf{M G}+\mathbf{A S}$ & $1 / k_{350} \mathrm{~nm}(\mathrm{~min})$ \\
\hline Trial 1 & 12.6 \\
\hline Trial 2 & 18.8 \\
\hline Trial 3 & 14.7 \\
\hline Avg. & $15.4 \pm 3.2$ \\
\hline Atm. Half-life & $12 \pm 3$ \\
\hline MG+MA & $1 / k_{350 \mathrm{~nm}}(\mathrm{~min})$ \\
\hline Trial 1 & 41.9 \\
\hline Trial 2 & 59.2 \\
\hline Trial 3 & 44.0 \\
\hline Avg. & $47.3 \pm 9.5$ \\
\hline Atm. Half-life & $39 \pm 8$ \\
\hline
\end{tabular}


III. PARAFAC

a. Excitation and Emission Spectra of PARAFAC Components

a) $\mathrm{MG}+\mathrm{AS}$
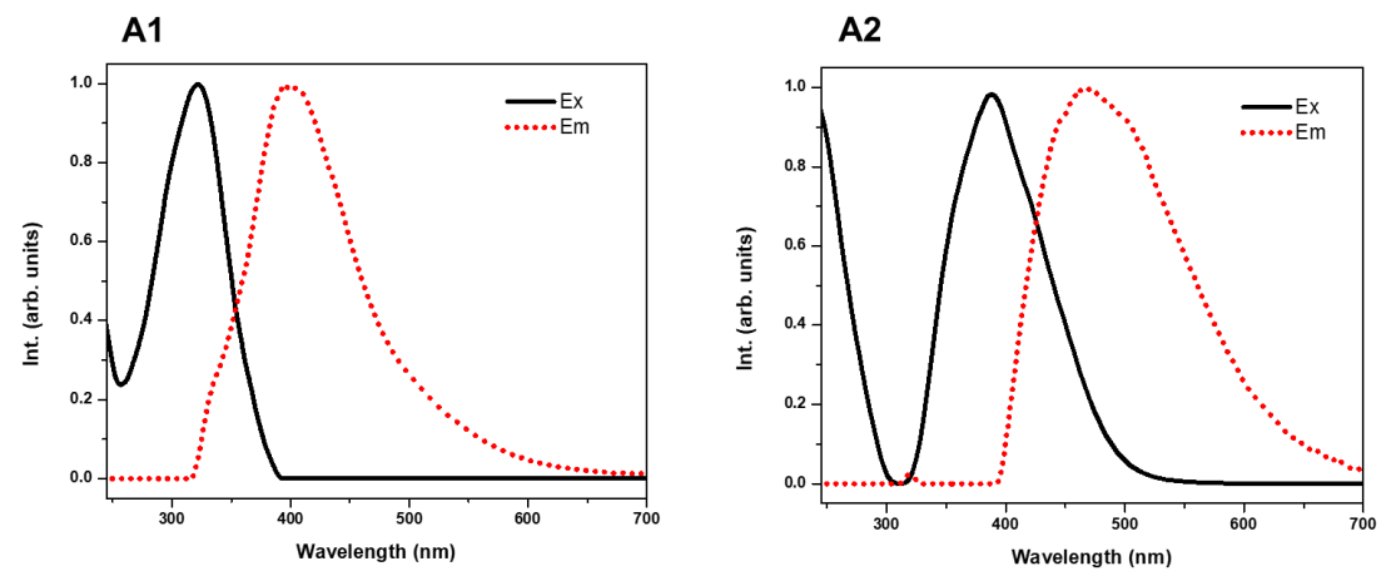

b) MG+MA
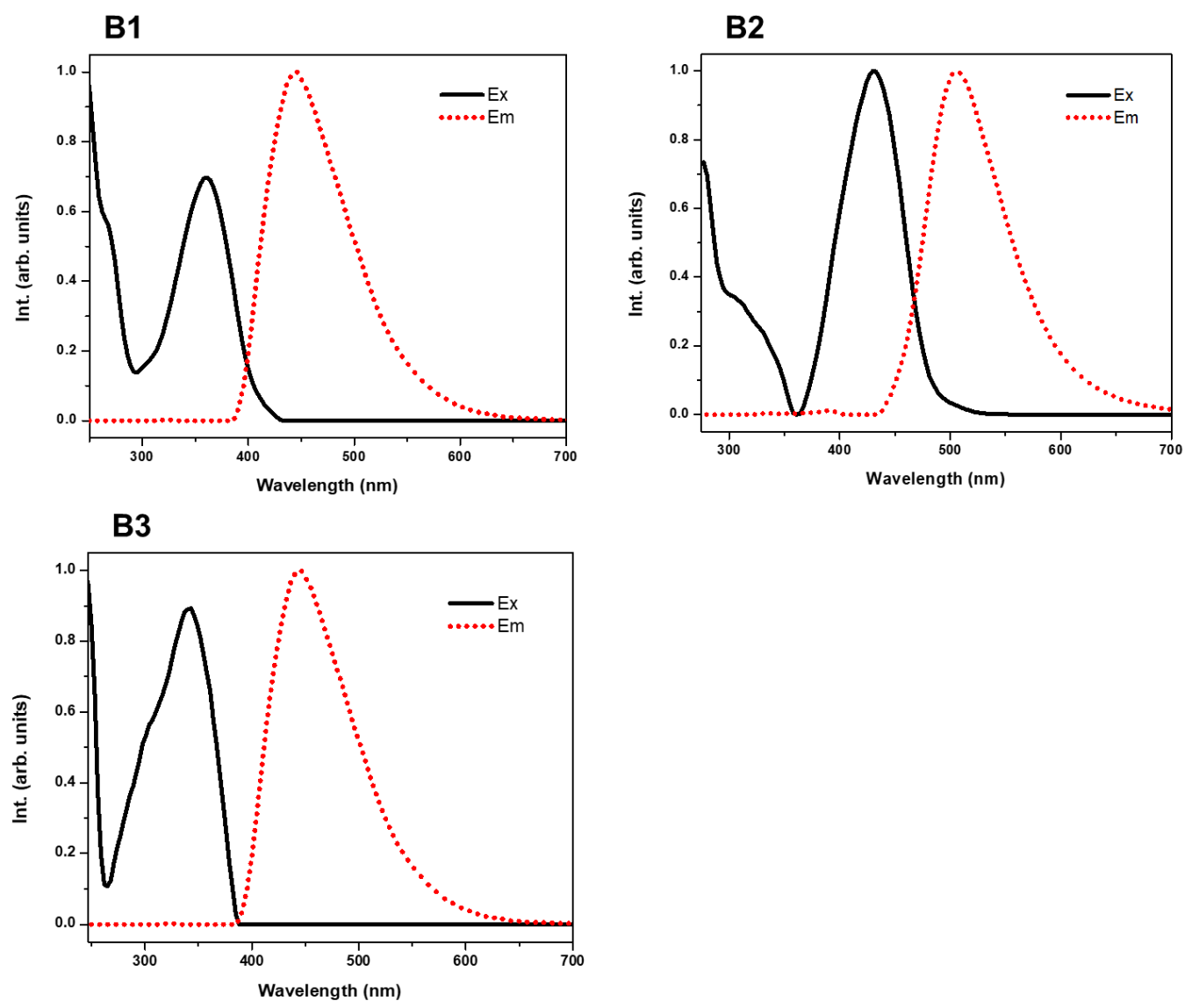

Figure S1. Excitation and Emission Spectra of PARAFAC Components in a) MG+AS and b) MG+MA BrC Samples 
b. Fluorophore Atmospheric Photolytic Half-lives

Table S2. Atmospheric Photolytic Half-lives of Component Fluorophores in MG+AS and MG+MA BrC Samples

\begin{tabular}{cccc}
\hline MG+AS & $\mathbf{1} / \boldsymbol{k}_{\mathbf{A} \mathbf{1}}(\mathbf{m i n})$ & $\mathbf{1} / \boldsymbol{k}_{\mathbf{A} 2}(\mathbf{m i n})$ & \\
Trial 1 & 16.4 & 8.5 & \\
Trial 2 & 17.9 & 10.0 & \\
Trial 3 & 21.4 & 10.7 & \\
Avg. & $18.6 \pm 2.6$ & $9.7 \pm 1.1$ & \\
Half-life & $15 \pm 2$ & $8 \pm 1$ & \\
\hline MG+MA & $\mathbf{1} / \boldsymbol{k}_{\mathbf{B} \mathbf{1}}(\mathbf{m i n})$ & $\mathbf{1} / \boldsymbol{k}_{\mathbf{B} \mathbf{2}}(\mathbf{m i n})$ & $\mathbf{1} / \boldsymbol{k}_{\mathbf{B} \mathbf{3}}(\mathbf{m i n})$ \\
\hline Trial 1 & 23.3 & 27.8 & 53.3 \\
Trial 2 & 21.1 & 26.8 & 21.2 \\
Trial 3 & 36.8 & 51.2 & 38.1 \\
Avg. & $27.1 \pm 8.5$ & $35.3 \pm 13.8$ & $37.5 \pm 16.1$ \\
Atm. Half-life & $23 \pm 7$ & $29 \pm 11$ & $31 \pm 13$ \\
\hline \hline
\end{tabular}


IV. Proposed Structures from Mass Spectra

Table S3. Proposed structures for MG+AS BrC based on chemical mechanism from previous references. Masses are detected as $\mathrm{M}+\mathrm{H}^{+}$and $\mathrm{M}+$ in methylated amine species

\begin{tabular}{lllc} 
Measured $\mathrm{m} / \mathrm{z}$ & Formula & Structure & Reference \\
\hline $83,97,111$ & $\begin{array}{l}\mathrm{C}_{4} \mathrm{H}_{6} \mathrm{~N}_{2} \\
\mathrm{C}_{5} \mathrm{H}_{9} \mathrm{~N}_{2}\end{array}$ & $\mathrm{C}_{6} \mathrm{H}_{11} \mathrm{~N}_{2}$ & De Haan, et al. EST 2011 \\
109 & $\mathrm{C}_{6} \mathrm{H}_{8} \mathrm{~N}_{2}$ & Hawkins, et al. ACP 2019 \\
111,125 & $\mathrm{C}_{5} \mathrm{H}_{6} \mathrm{~N}_{2} \mathrm{O}$ & De Haan, et al. EST 2011 \\
& $\mathrm{C}_{6} \mathrm{H}_{8} \mathrm{~N}_{2} \mathrm{O}$ &
\end{tabular}

181

$\mathrm{C}_{9} \mathrm{H}_{12} \mathrm{~N}_{2} \mathrm{O}_{2}$

Hawkins, et al. ACP 2019

234, 306, 378

$\mathrm{C}_{12} \mathrm{H}_{15} \mathrm{~N}_{3} \mathrm{O}_{2}$
$\mathrm{C}_{15} \mathrm{H}_{19} \mathrm{~N}_{3} \mathrm{O}_{4}$
$\mathrm{C}_{18} \mathrm{H}_{23} \mathrm{~N}_{3} \mathrm{O}_{6}$

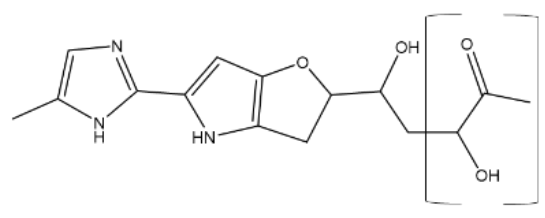

Lin, et al. ES\&T 2015

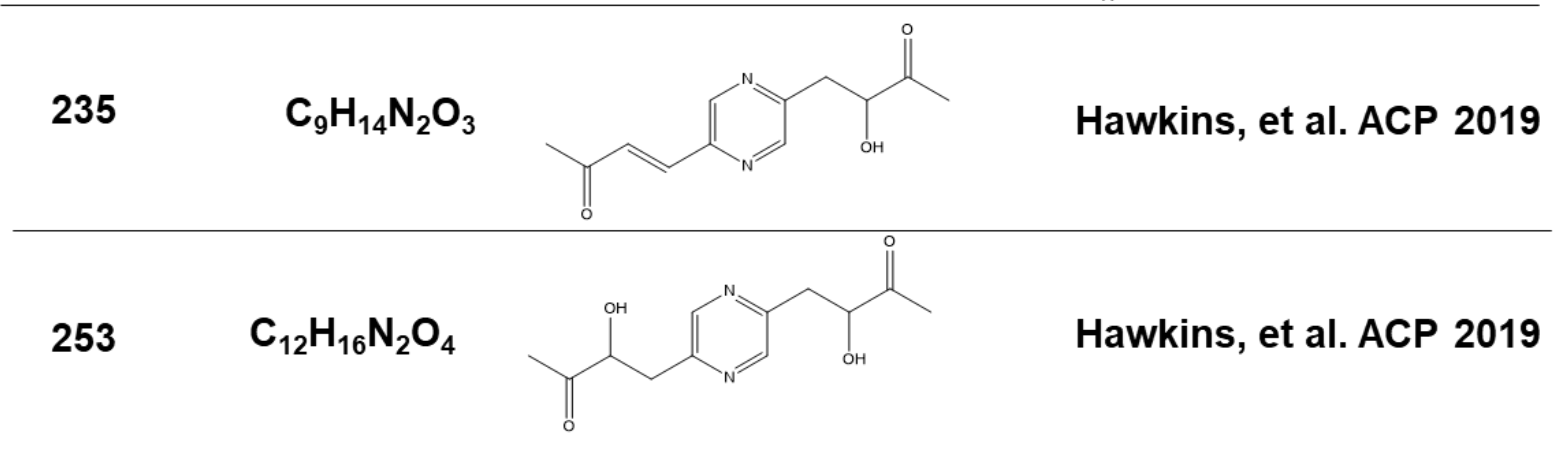

272,344

$\mathrm{C}_{11} \mathrm{H}_{17} \mathrm{~N}_{3} \mathrm{O}_{5}$

$\mathrm{C}_{14} \mathrm{H}_{21} \mathrm{~N}_{3} \mathrm{O}_{7}$

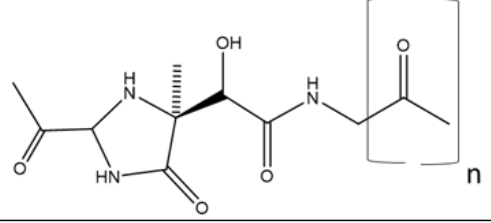

De Haan, et al. GRL 2009

289

$\mathrm{C}_{15} \mathrm{H}_{16} \mathrm{~N}_{2} \mathrm{O}_{4}$

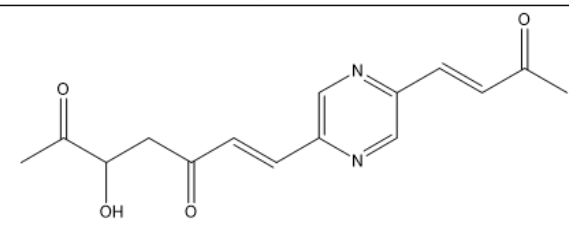

Hawkins, et al. ACP 2019 
Table S4. Proposed structures for MG+MA BrC based on chemical mechanism from previous references. Masses are detected as $\mathrm{M}+\mathrm{H}^{+}$and $\mathrm{M}+$ in methylated amine species

\begin{tabular}{|c|c|c|c|}
\hline Measured $m / z$ & Formula & Structure & Reference \\
\hline 97,111 & $\begin{array}{l}\mathrm{C}_{5} \mathrm{H}_{9} \mathrm{~N}_{2} \\
\mathrm{C}_{6} \mathrm{H}_{11} \mathrm{~N}_{2}\end{array}$ & & De Haan, EST et al 2011 \\
\hline $111,125,139,153$ & $\begin{array}{l}\mathrm{C}_{5} \mathrm{H}_{6} \mathrm{~N}_{2} \mathrm{O} \\
\mathrm{C}_{6} \mathrm{H}_{8} \mathrm{~N}_{2} \mathrm{O} \\
\mathrm{C}_{7} \mathrm{H}_{10} \mathrm{~N}_{2} \mathrm{O} \\
\mathrm{C}_{9} \mathrm{H}_{12} \mathrm{~N}_{2} \mathrm{O}\end{array}$ & & De Haan, EST et al 2011 \\
\hline 167 & $\mathrm{C}_{9} \mathrm{H}_{14} \mathrm{~N}_{2} \mathrm{O}$ & & De Haan, EST et al 2018 \\
\hline 171 & $\mathrm{C}_{8} \mathrm{H}_{14} \mathrm{~N}_{2} \mathrm{O}_{2}$ & & De Haan, et al GRL 2009 \\
\hline
\end{tabular}

235

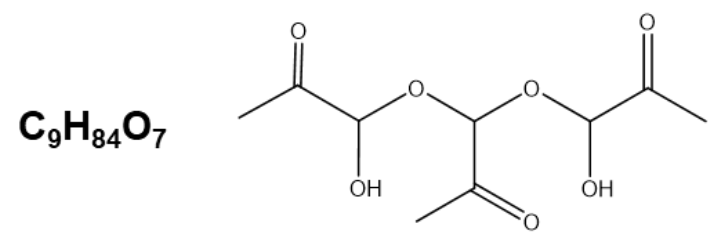

Sareen, et al. ACP 2010

271

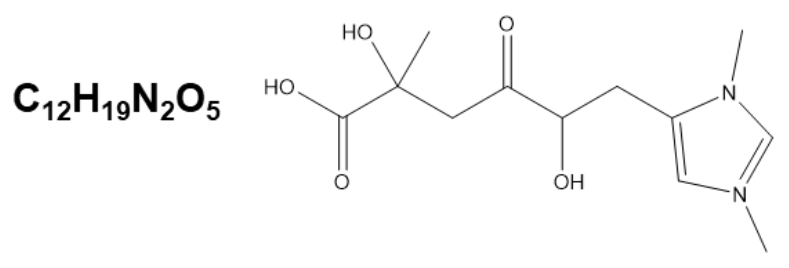

De Haan, et al. ACS E\&S Chem 2019

*See Refs. ${ }^{13-16}$ below for Tables S3 and S4 


\section{References}

(1) Laskin, A.; Laskin, J.; Nizkorodov, S. A. Chemistry of Atmospheric Brown Carbon. Chem. Rev. 2015, 115 (10), 4335-4382.

(2) Aiona, P. K.; Lee, H. J.; Leslie, R.; Lin, P.; Laskin, A.; Laskin, J.; Nizkorodov, S. A.

Photochemistry of Products of the Aqueous Reaction of Methylglyoxal with Ammonium Sulfate. ACS Earth and Space Chemistry 2017, 1 (8), 522-532.

(3) Zhao, R.; Lee, A. K. Y.; Abbatt, J. P. D. Investigation of Aqueous-Phase Photooxidation of Glyoxal and Methylglyoxal by Aerosol Chemical Ionization Mass Spectrometry: Observation of Hydroxyhydroperoxide Formation. J. Phys. Chem. A 2012, 116 (24), 62536263.

(4) Zhao, R.; Lee, A. K. Y.; Huang, L.; Li, X.; Yang, F.; Abbatt, J. P. D. Photochemical Processing of Aqueous Atmospheric Brown Carbon. Atmos. Chem. Phys. 2015, 15 (11), 6087-6100.

(5) Powelson, M. H.; Espelien, B. M.; Hawkins, L. N.; Galloway, M. M.; De Haan, D. O. Brown Carbon Formation by Aqueous-Phase Carbonyl Compound Reactions with Amines and Ammonium Sulfate. Environ. Sci. Technol. 2014, 48 (2), 985-993.

(6) Haan, D. O. D.; Tolbert, M. A.; Jimenez, J. L. Atmospheric Condensed-Phase Reactions of Glyoxal with Methylamine. Geophysical Research Letters 2009, 36 (11).

(7) Marrero-Ortiz, W.; Hu, M.; Du, Z.; Ji, Y.; Wang, Y.; Guo, S.; Lin, Y.; Gomez-Hermandez, M.; Peng, J.; Li, Y.; Secrest, J.; Zamora, M. L.; Wang, Y.; An, T.; Zhang, R. Formation and Optical Properties of Brown Carbon from Small $\alpha$-Dicarbonyls and Amines. Environ. Sci. Technol. 2019, 53 (1), 117-126.

(8) Hawkins, L. N.; Welsh, H. G.; Alexander, M. V. Evidence for Pyrazine-Based Chromophores in Cloud Water Mimics Containing Methylglyoxal and Ammonium Sulfate. Atmospheric Chemistry and Physics 2018, 18 (16), 12413-12431.

(9) Grace, D. N.; Sharp, J. R.; Holappa, R. E.; Lugos, E. N.; Sebold, M. B.; Griffith, D. R.; Hendrickson, H. P.; Galloway, M. M. Heterocyclic Product Formation in Aqueous Brown Carbon Systems. ACS Earth Space Chem. 2019.

(10) Aiona, P. K.; Lee, H. J.; Lin, P.; Heller, F.; Laskin, A.; Laskin, J.; Nizkorodov, S. A. A Role for 2-Methyl Pyrrole in the Browning of 4-Oxopentanal and Limonene Secondary Organic Aerosol. Environ. Sci. Technol. 2017, 51 (19), 11048-11056. 
(11) De Haan, D. O.; Hawkins, L. N.; Welsh, H. G.; Pednekar, R.; Casar, J. R.; Pennington, E. A.; de Loera, A.; Jimenez, N. G.; Symons, M. A.; Zauscher, M.; Pajunoja, A.; Caponi, L.; Cazaunau, M.; Formenti, P.; Gratien, A.; Pangui, E.; Doussin, J.-F. Brown Carbon Production in Ammonium- or Amine-Containing Aerosol Particles by Reactive Uptake of Methylglyoxal and Photolytic Cloud Cycling. Environ. Sci. Technol. 2017, 51 (13), 74587466.

(12) Nguyen, T. B.; Lee, P. B.; Updyke, K. M.; Bones, D. L.; Laskin, J.; Laskin, A.;

Nizkorodov, S. A. Formation of Nitrogen- and Sulfur-Containing Light-Absorbing Compounds Accelerated by Evaporation of Water from Secondary Organic Aerosols. Journal of Geophysical Research: Atmospheres 2012, 117 (D1).

(13) De Haan, D. O.; Hawkins, L. N.; Kononenko, J. A.; Turley, J. J.; Corrigan, A. L.; Tolbert, M. A.; Jimenez, J. L. Formation of Nitrogen-Containing Oligomers by Methylglyoxal and Amines in Simulated Evaporating Cloud Droplets. Environ. Sci. Technol. 2011, 45 (3), 984-991.

(14) De Haan, D. O.; Tapavicza, E.; Riva, M.; Cui, T.; Surratt, J. D.; Smith, A. C.; Jordan, M.C.; Nilakantan, S.; Almodovar, M.; Stewart, T. N.; de Loera, A.; De Haan, A. C.; Cazaunau, M.; Gratien, A.; Pangui, E.; Doussin, J.-F. Nitrogen-Containing, LightAbsorbing Oligomers Produced in Aerosol Particles Exposed to Methylglyoxal, Photolysis, and Cloud Cycling. Environ. Sci. Technol. 2018, 52 (7), 4061-4071.

(15) Sareen, N.; Schwier, A. N.; Shapiro, E. L.; Mitroo, D.; McNeill, V. F. Secondary Organic Material Formed by Methylglyoxal in Aqueous Aerosol Mimics. Atmospheric Chemistry and Physics 2010, 10 (3), 997-1016.

(16) De Haan, D. O.; Pajunoja, A.; Hawkins, L. N.; Welsh, H. G.; Jimenez, N. G.; De Loera, A.; Zauscher, M.; Andretta, A. D.; Joyce, B. W.; De Haan, A. C.; Riva, M.; Cui, T.; Surratt, J. D.; Cazaunau, M.; Formenti, P.; Gratien, A.; Pangui, E.; Doussin, J.-F. Methylamine's Effects on Methylglyoxal-Containing Aerosol: Chemical, Physical, and Optical Changes. ACS Earth Space Chem. 2019. 